\title{
Index rerum ad Vol. 144
}

\section{Confecit F. Ammann, Geneve}

(B) = Buchbesprechungen - Livres nouveaux - Book reviews. $(\mathrm{R})=$ Referate von Vorträgen, die an anderer Stelle ausführlich erschienen sind oder erscheinen werden. Einzelheiten werden in diesem Index rerum nicht berücksichtigt. - Rapports de conferences qui ont été (ou seront) publiées ailleurs in extenso. Cet Index rerum ne contient pas de details. - Abstract $\mathrm{s}$ of lectures that have been (or will be) published in extenso elsewhere. Details are not included in this index rerum. $(\mathrm{V})=$ Vortrag - Communication - Report.

Ablatio retinae, v. Electroretinogram

Acrylatlinsen; Untersuchungen über die Toleranz von Acrylatlinsen bei aphakischen Kaninchen, Ill

Action potential of the ciliary muscle, 323

Acuité visuelle, v. Visual acuity

Adaptation; the mechanism of dark adaptation, 255 (V)

Adaptation à Гobscurité, v. Adaptation

Albinism, ocular, v. Diagnosis

Albright's syndrome; cataract associated with Albright's syndrome (osteitis fibrosa), 371

Alloxan diabetes in the pregnant mouse (Acta Ophthalmologica Suppl. 68) , 397 (B)

Amblyopia; attention and amblyo-pia, an approach by way of elec-troencephalography, 298 (V)

Anabolic steroids, v. Diabetic retino-pathy

Anatomie des Sehapparates, v. Ophthalmology

Anatomy of visual system, v. Ophthalmology

Angle irido-cornéen, v. Chamber angle surgery, Trabecula

Angle of anterior chamber, v. Chamber angle surgery, Trabecula

Année thérapeutique et clinique en ophtalmologie (Vol. 11), 396 (B)

Anterior chamber; variation in depth of the anterior chamber of the eye and variation in intraocular pressure, $259(\mathrm{~V})$

Anterior chamber, v. Vorderkammer

Antibiotica et Chemotherapeutica, v. Anabolic steroids, Decadura-bolin, Diamox, Penicillins,

Sofra-mycin

Applanations-Tonometrie; Applana-tionstonometrie an liegender Ver-suchsperson, 93

Applanations-Tonometrie, v. Netz-hautzentralarterie

Aqueous flow; on the connection between aqueous flow and flare, 153

Aqueous veins, 310 (B)

Atrophia optica, v. Leber's disease

Auge, v. Eye

Augenabschnitt, hinterer, v. Tumors

Augenarzt (Vol. 4), 395 (B)

Augenbinnendruck, v. Anterior chamber, Applanations-Tonometrie, Intraocular pressure,

Maklakoff-Tonometer, Rigiditäts-bestimmung, Rigidity, Tonograms 
Augenhintergrundsveränderungen, v. Albinism, ocular, Choroiditis disseminata acuta, Degeneratio hyaloideo-retinalis hereditaria, Diabetic retinopathy, Leber's disease

Barrière hémato-oculaire, v. Vitreous

body Begulachtung; das Auge in Begut-

achtung und Gesetzgebung, 229 (B) Blood-aqueous barrier, v. Vitreous

body Blut-Kammerwasserschranke,

v. Vitreous body Book reviews, 151 (B), 229-230 (B),

310 (B), 395-397 (B), 465 (B)

Index rerum ad Vol. 144

467

Brain, v. Verkalkungen Buchbesprechungen, 151 (B),

229-230 (B), 310 (B), 395-397 (B),

465 (B)

Calcifications intracraniennes, v. Verkalkungen

Calcium deposits; treatment of calcium deposits in the cornea by irrigation and application of

E.D.T.A., 451 (V)

Camera anterior, v. Anterior chamber, Vorderkammer

Canaliculitis; chronic canaliculitis caused by Fusobacterium Plaut-Vincenti and Borrelia

Vincentii, 309 (V)

Cat; the effect of monocular and binocular photic stimulation upon the electric potentials of retina and visual cortex of the anaesthetized cat, 257 (V)

Cataract; clinical observations on 1000 operations for cataract, 375

Cataract associated with Albright's syndrome, 371

Central retinal artery; results of early treatment in a case of spasm of the central retinal artery, $433(\mathrm{~V})$

Chamber angle surgery, $402(\mathrm{~V})$

Chambre antérieure, v. Anterior chamber, Vorderkammer

Chat, v. Cat

Chemotherapeutica et Antibiotica, v. Anabolic steroids, Decadura-bolin, Diamox, Penicillins,

Sofra-mycin

Chloroquine, v. Nivaquine

Choriocapillaire, v. Microscope électronique

Choroiditis disseminata acuta, treatment by Prednison, 291 (V)

Ciliary body, v. Melanoblastoma, Tumour

Ciliary muscle; the action potential of the ciliary muscle, 323

Congress, v. Netherlands Ophthalmo-logical Society, Ophthalmological Society of the United

Kingdom

Cornea, v. Calcium deposits, Herpes simplex corneae, Megalocornea

Corps ciliaire, v. Melanoblastoma, Tumour

Corpus vitreum, v. Vitreous body Cristallin, v. Acrylatlinsen, Linse

Dark adaptation, mechanism, 255 (V)

Decadurabolin, v. Diabetic retino-pathy

Décollement de la rétine, v. Electro-relinogram

Degeneratio hyaloideo-retinalis hereditaria, 458 (V)

Demonstration of cases, 276-283 (V), 420-425 (V) 
Demonstration of films, 404 (V)

Depth of anterior chamber, v. Anterior chamber

Detachment of retina, v. Electro-retinogram

Diabetes; Alloxan diabetes in the pregnant mouse (Acta Ophthalmo-logica Suppl. 68) , 397 (B)

Diabetic retinopathy; periodical integration of retinal responses following repetitive light

stimuli- a method for the demonstration of minor changes in retinal activity in diabetic

retinopathy, $403(\mathrm{~V})$

-; the treatment of diabetic retinopathy with anabolic steroids, $403(\mathrm{~V})$

-; treatment of diabetic retinopathy with Nandrolone-Decanoate (Decadurabolin), 346

Diagnose, v. Strabismus, Uveitis, Verkalkungen

Diagnosis; a diagnostic difficulty solved by co-operative effort, 296 (V)

Diagnosis, v. Tumors

Diamox, v. Scintillating scotoma

Differenzwert, v. Rigiditätsbestim-mung

Documenta Ophthalmologica (Vol. 15), 396 (B)

Druck, v. Netzhautzentralarterie

Dunkeladaptation, v. Adaptation

Dynamometrie, v. Ophthalmo-dynamometrie

Eleclroencephalography,

v. Amblyopia Electromyography, v. Elektromyo-

graphie, Stilling-Türk-Duane

syndrome

468

Index rerum ad Vol. 144

Electrophysiology, v. Ciliary muscle

Electroretinography, clinical, 310 (B)

Electroretinogram; an end-effect in the human E.R.G., 451 (V)

-; the clinical electroretinogram in detachment of the retina (Acta Ophthalmologica Suppl. 64), 151 (B)

Electroretinogram, v. Gat, Diabetic retinopathy

Electrorétinogramme; etude électro-rétinographíque de Гæil humain énucléé, 126

Electrotonometer, v. Tonograms

Elektromyographie, v. Myopathie, oculäre

Elektroretinogramm, v. Electro ...

Entzündungen, v. Inflammations

Epidermoid, supraselläres (Bemer-kungen zu der neurologischen, ophthalmologischen und röntgeno-logischen Symptomatologie), 231

ERG, v. Electroret. .., Electrorét...

Exophthalmos, endocrine (Acta Ophthalmologica Suppl. 65), 151 (B)

Experimentelle Forschung, v. Acry-latlinsen, Diabetes, Electroretinogram, Exophthalmos, endocrine, Lacrimal gland, Penicillins, Retina, Soframycin, Trabecula, Vitreous body

Expertise, v. Begutachtung

Eye; attenuation curves of the human eye under normal and pathological conditions, 451 (V)

Eye, malformations, v. Augenarzt, Diabetes

Eye, muscles, v. Listing's law 
Films, demonstrations, 404 (V)

Flare, v. Aqueous flow

Fîicker-Electroretinogram, v. Diabetic retinopathy

Framycetin, v. Soframycin

Fuchs' heterochromia, v. Reckling-hausen's disease

Fundus, v. Albinism, ocular, Cho-roiditis disseminata acuta, De-generatio hyaloideo-retinalis hereditaria, Diabetic retinopathy, Leber's disease

G11/8fäße, v. Aqueous veins, Central retinal artery, Netzhautzentralarterie Gehirn, v.

Verkalkungen Gesellschaft fur Ophthalmologie,

v. Ophthalmological Society Gesichtssinn; Lehrbuch der Physio-logie: Der Gesichtssinn (2.

Aufl.), 230 (B) Glande lacrymale, v. Lacrimal gland Glaskörper, v. Vitreous body Glaucoma; therapeutic results in

primary glaucoma, 395 (B) Glaukom, v. Iridenkleisis Golgi apparatus in the rabbit lacrimal gland, 165 Gonioscopy; slit-lamp gonioscopy,

151 (B) Graft, corneal, v. Trephine Greffe cornéenne, v. Trephine

Heracles; Marcus Ulpius Heracles,

the first ophthalmologist at Nij-

megen, 451 (V) Heredity, v. Leber's disease Herpes simplex corneae, statistical

investigation on the occurrence,

405 (V) Heterochromia Fuchs, v. Reckling-

hausen's disease Histochemistry, v. Retina Histologie, v. Rétine, Lacrimal gland Historie, v.

Heracles, Tonometer Hornhaut, v. Calcium deposits, Herpes simplex corneae, Megalocornea

Hornhautplastik, v. Trephine Humeur aqueuse, v. Aqueous flow,

Aqueous veins, Uveitis Humor aqueus, v. Aqueous flow,

Aqueous veins, Uveitis Hyaloideo-retinalis; degeneratio

hyaloideo-retinalis hereditaria,

$458(\mathrm{~V})$

Inflammations; endogenous inflammations of the uveal tract, 310 (B)

In memoriam W. P. C. Zeeman, 254 (V)

Instruments, v. Trephine

Intraocular pressure; variation in depth of the anterior chamber of the eye and variation in intraocular pressure, $259(\mathrm{~V})$

Index rerum

ad Vol. 144

469

Intraocular pressure, v. Anterior chamber, Applanations-Tono-metrie, Maklakoff-Tonometer, Rigiditätsbestimmung, Rigidity, Tonograms

Intraokularer Druck, v. Anterior chamber, Applanations-Tono-metrie, Intraocular pressure, Maklakoff-Tonometer, Rigiditätsbestimmung, Rigidity, Tonograms

Iridectomy; central capsulary irid-ectomy, 302 (V)

Iridenkleisis; klinische Erfahrungen mit der Iridenkleisis nach dem Verfahren von L. u. R.

Weekers, 81

Iridoschisis, 97

Iris cyst, v. Melanoblastoma

Iris, v. Iridectomy, Tumour 
Kammerwasser, v. Aqueous flow, Aqueous veins, Uveitis

Kammerwinkel, v. Chamber angle surgery, Trabecula

Kaninchen, v. Acrylatlinsen, Lacrimal gland, Penicillins, Retina, Soframycin, Vitreous body

Katarakt, v. Cataract

Katze, v. Cat

Keratoplasty, v. Trephine

Kollapsdruck, v. Netzhautzentral-arterie

Kongresse, v. Ophthalmological Society

Lacrimal gland; the Golgi apparatus in the rabbit lacrimal gland, 165

Lampe à fente, v. Gonioscopy

Lapin, v. Acrylatlinsen, Lacrimal gland, Penicillins, Retina, Soframycin, Vitreous body

Leber's disease; an investigation of the occurrence of Leber's disease in the Netherlands, 415

(V)

-; optical correlations in Leber's optic atrophy, 426 (V)

-; observations on the heredity of Leber's disease, 446 (V)

Leber's optic atrophy, v. Leber's disease

Lens, v. Acrylatlinsen, Linse

Libri, 151 (B), 229-230 (B), 310 (B), 395-397 (B), 465 (B)

Linse; eine neue Einrichtung zur Messung der Tiefe der mensch-lichen Vorderkammer und der

Dicke der Linse, 49

Linse, v. Acrylatlinsen

Listing's law; ophthalmotropic measurements: deliberations about Listing's law, 288 (V)

Livres nouveaux, 151 (B), 229-230 (B), 310 (B), 395-397 (B), 465 (B)

Maklakoff-Tonometer; zur Kalibrie-rung des Maklakoff-Tonometers (vorläufige Mitteilung), 391

Maladie de Leber, v. Leber's disease

Maladie de Recklinghausen, v. Reck-linghausen's disease

Malformations oculaires, v. Augen-arzt, Diabetes

Maus, v. Diabetes

Megalocornea, 304 (V)

Melanoblastoma; the concurrence of an iris cyst and melanoblastoma of the ciliary body, 284 (V)

Methode, v. Applanations-Tono-metrie, Linse, Maklakoff-Tonometer, Netzhautzentralarterie, Ophthalmodynamometrie, Strabismus, Trephine, Ultraschall, Visual acuity, Vorderkammer Microscope électronique; etude des capillaires de la rétine et de la choriocapillaire au microscope électronique, 1

Migraine ophtalmique, v. Scintillating scotoma

Migräne, ophthalmische, v. Scintillating scotoma

Mißbildungen des Auges, v. Augen-arzt, Diabetes

Mouse, v. Diabetes

Müller-Electrotonometer, v. Tonograms

Muscle ciliaire, v. Ciliary muscle

Muskeln, v. Listing's law

Myopathie, oculäre; über die chro-nisch progressive oculäre Myopathie, 29

Netherlands Ophthalmological Society, 146th Meeting (1960), 252-309 (V); 147th Meeting (1961), 400-444 (V); 148th Meeting (1961), 446-465 (V)

470 
Index rerum

ad Vol. 144

Netzhaut, v. Retina, Rétine Netzhautablösung, v. Electroretino-

gram Netzhautzentralarterie; direkte Be-

slimmung des Kollapsdruck.es der

Netzhautzentralarterie beim Men-

schen, 62 Neuritis retrobulbaris, v. L·eber's

disease Nivaquine; retinopathy after

Nivaquine treatment, $438(\mathrm{~V})$

Ocular tension, v. Anterior chamber, Applanations-Tonometrie, Intraocular pressure, Maklakoff-

Tono-meter, Rigiditätsbestimmung, Rigidity, Tonograms

OEil, v. Eye

(Eil humain énucléé, etude électro-rétinographique, 126

Operation, v. Cataract, Chamber angle surgery, Iridectomy, Iriden-kleisis, Staphyloma,

Strabismus, Surgery, Tumour

Ophtalmologie; Гannée thérapeu-tique et clinique en ophtalmologie (Vol. 11), 396 (B)

-; Encyclopédie médico-chirurgicale: Ophtalmologie, 230 (B)

Ophthalmic migraine, v. Scintillating scotoma

Ophthalmodynamometrie, 310 (B)

Ophthalmologica; Documenta

Ophthalmologica (Vol. 15), 396 (B)

Ophthalmological Society, Netherlands, 146th Meeting (1960), 252-309 (V); 147th Meeting (1961), 400-444 (V); 148th Meeting (1961), 446-465 (V)

Ophthalmological Society of the United Kingdom, 82nd congress (1962), 78-80 (R)

Ophthalmological Society of the United Kingdom, transactions (1961) (Vol. 81), 397 (B)

Ophthalmology; system of ophthalmology (Vol. 2), 229 (B)

Ophthalmoplegia externa chronica progressiva inlernuclearis, v. Myopalhie, oculäre

Ophthalmolropic measurements: deliberations about Listing's law, 288 (V)

Optic atrophy, v. L·eber's disease Optikusalrophie, v. L·eber's disease Optokinetic nystagmus,

v. Visual

acuity Orbita; über das nicht-chromaffine

Paraganglíom der Orbita, 103 Osteitis fibrosa, v. Albright's

syndrome

Paragangliom, nicht-chromaffines, der Orbita, 103

Penicillins; the penetration of several new Penicillins into the tissues of the eye, 316

Physiologie, v. Gesichtssinn

Plaut-Vincent-canaliculítis, v. Canaliculitis

Posterior segment of the bulb, v. Tumors

Prednison, v. Choroiditis dis-seminata acuta

Pression oculaire, v. Anterior Chamber, Applanations-Tonometrie, Intraocular pressure,

Maklakoff-Tonometer, Rigiditätsbestimmung, Rigidity, Tonograms

Proceedings, 78-80 (R)

Profondeur de la chambre anté-rieure, v. Anterior chamber

Pupil, artificial, v. Iridectomy

Rabbit, v. Acrylatlinsen, L-acrimal gland, Penicillins, Retina, Sofra-mycin, Vitreous body 
Radioactive phosphorus, v. Tumors

Recherches expérimentales, v. Acrylatlinsen, Diabetes, Electroretino-gram, Exophthalmos, endocrine, L-acrimal gland, Penicillins, Retina, Soframycin, Trabecula, Vitreous body Recklinghausen's disease; Fuchs' heterochromia in a patient with Recklinghausen's disease, 409

(V)

Regenbogenhaut, v. Iris

Retina; protein metabolism of rabbit retina in vitro studied by means of high resolution autoradiography, 13

-; radioautographic investigations on nucleic acids and protein metabolism of the retina in vitro, 141

Index rerum

ad Vol. 144

471

Rétine; etude des capillaires de la rétine et de la choriocapillaire au microscope électronique, 1

Retino-cortical time, v. Cat

Retinopathia diabetica, v. Diabetic retinopathy

Retinopathy after Nivaquine treatment, 438 (V)

Retrobulbärneuritis, v. Leber's disease

Rigiditätsbestimmung; intraokularer Druck, Körperlage und Rigiditätsbestimmung, 57

Rigidity; calculations on scleral rigidity, 264 (V)

Rigidity, v. Tonograms

Schielen, v. Stilling-Türk-Duane

syndrome, Strabismus Schwarzer-Electrotonomeler,

v. Tonograms Schwarzer-Eleklrotonometer,

v. Rigiditätsbestimmung Scintillating scotoma; Diamox in

the treatment of scintillating

scotoma, 341 Scleral rigidity, calculations, 264 (V) Segment postérieur de Гæíl,

v. Tumors Sehschärfe, v. Visual acuity Sens visuel, v. Gesichtssinn Sitzungsberichte, 78-80 (R)

Slit-lamp gonioscopy, 151 (B) Sociétés, comples rendus, 78-80 (R) Societies, v.

Ophthalmological

Society Society transactions, $78-80(\mathrm{R})$ Soframycin; intracameral injection

of Framycetin (Soframycin), 311 Souris, v. Diabetes Spaltlampe, v. Gonioscopy Spasm of the

central retinal artery,

results of early treatment in a

case, 433 (V) Squint, v. Stilling-Türk-Duane

syndrome, Strabismus Staphyloma; the surgical treatment

of post-traumatic intercalary

staphyloma, 299 (V) Statistische Auswertung, v. Cataract,

(ílaucoma, Herpes simplex cor-

neae, Iridenkleisis, Strabismus

Stilling-Türk-Duane syndrome; is the Stilling-Türk-Duane syndrome an independent pathological entity?, 199

Strabismus; exakte Strabismusdia-gnostik bei V2-3jährigen Kindern mit einem einfachen

Verfahren, dem «Durchleuchlungstest», 184

-; the results of operative treatment of convergent strabismus, 306 (V) 
Strabismus, v. Stilling-Türk-Duane syndrome

Surgery; demonstration of some surgical and technical modifications, 452 (V)

Survey, v. Begutachlung

Tarsitis tuberculosa, 175

Tension oculaire, v. Pression oculaire

Therapie, v. Calcium deposits, Cataract, Central retinal artery, Choroiditis disseminata acuta,

Diabetic retinopathy, Herpes simplex corneae, Leber's disease, Scintillating scotoma,

Staphyloma, Strabismus, Tumour

Tiefe der Vorderkammer, v. Anterior chamber

Tonograms; comparative calculation of tonograms, 268 (V)

Tonometer; über einige sehr alle Tonometer, 221

Tonometrie, v. Applanalions-Tono-melrie, Maklakoff-Tonometer, Netzhautzentralarterie,

Rigiditätsbestimmung

Tonometry, v. Rigidity, Tonograms

Trabecula; the innervation of the corneo-scleral trabecula, 455 (V)

Tränendrüse, v. Lacrimal gland

Tränenkanalentzündung, v. Canali-culitis

Transactions of the Ophthalmological Society of the United Kingdom (Vol. 81), 397 (B)

Trauma, v. Staphyloma

Trepan, v. Trephine

Trephine; a helix trephine for penetrating keratoplasty, 385

Tumoren, v. Epidermoid, supra-selläres, Melanoblastoma, Orbita, Tumors, Tumour

472

Index rerum

ad Vol. 144

Tumors; diagnosis of tumors in the posterior part of the eye by P32, 400 (V)

Tumour; removal of a tumour from the iris and ciliary body by incision in the sclera, 295 (V)

Tyndall, v. Aqueous flow

Ultraschall; über die Möglichkeiten

einer Ultraschalluntersuchung in

der Augenheilkunde, 20 United Kingdom, v. Ophthal-

mological Society Untersuchungstechnik, v. Applana-

tions-Tonometrie, Maklakoff-

Tonometer, Netzhautzentral-

arterie, Strabismus, Ultraschall,

Visual acuity Uvea, v. Inflammations Uveitis; the importance of humor

aqueous analyses in the diagnosis

of uveitis, 244 Uveitis, v. Choroiditis disseminata

acuta

Vaisseaux, v. Aqueous veins, Central retinal artery, Netzhautzentral-arterie

Varia, 152, 398, 465

Vererbung, v. Leber's disease

Verhandlungsberichte, 78-80 (R)

Verkalkungen; endokranielle Verkal-kungen im Röntgenbild. Ihre Deu-tung und Bedeutung im Dienste der klinischen Hirndiagnostik, 645 (B) 
Versammlungsberichte, v. Verhandlungsberichte

Vessels, v. Aqueous veins, Central retinal artery, Netzhautzentral-arterie

Visual acuity; the objective

measurement of visual acuity, by arresting optokinetic nystagmus (Acta Ophthalmologica

Suppl.66), 395 (B)

Visual sense, v. Gesichtssinn

Vitreous body; osmotically induced change in the volume of the vitreous body causing protrusion of the ocular diaphragm, 263 (V)

Vorderkammer; eine neue Einrich-tung zur Messung der Tiefe der menschlichen Vorderkammer und der Dicke der Linse, 49

Vorderkammer, v. Anterior chamber

Zeeman, W. P. C, in memoriam,

2. 254 (V) Ziliarkörper

v. Melanoblastoma,

Tumour Ziliarmuskel

v. Ciliary muscle 